\title{
On The Structure of Competitive Societies
}

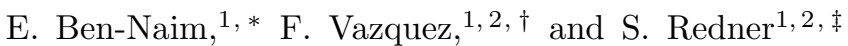 \\ ${ }^{1}$ Theoretical Division and Center for Nonlinear Studies, \\ Los Alamos National Laboratory, Los Alamos, New Mexico 87545 \\ ${ }^{2}$ Department of Physics, Boston University, Boston, Massachusetts, 02215 USA
}

\begin{abstract}
We model the dynamics of social structure by a simple interacting particle system. The social standing of an individual agent is represented by an integer-valued fitness that changes via two offsetting processes. When two agents interact one advances: the fitter with probability $p$ and the less fit with probability $1-p$. The fitness of an agent may also decline with rate $r$. From a scaling analysis of the underlying master equations for the fitness distribution of the population, we find four distinct social structures as a function of the governing parameters $p$ and $r$. These include: (i) a static lower-class society where all agents have finite fitness; (ii) an upwardly-mobile middle-class society; (iii) a hierarchical society where a finite fraction of the population belongs to a middle class and a complementary fraction to the lower class; (iv) an egalitarian society where all agents are upwardly mobile and have nearly the same fitness. We determine the basic features of the fitness distributions in these four phases.
\end{abstract}

PACS numbers: 87.23.Ge, 02.50.Ey, 05.40.-a, 89.65.Ef

\section{INTRODUCTION}

The emergence of class structure in society is a ubiquitous phenomenon in the biological and the social sciences [1, 2, 3, 4]. Social hierarchies have been widely observed in animal populations including insects [5], mammals [6, 7, 8], and primates [9], as well as human communities [10].

The possibility of quantitative modeling of social phenomena using concepts and techniques borrowed from the physical sciences is rapidly gaining appreciation. Examples of such modeling include the wealth distribution [1, 12], opinion dynamics 13, 14, 15], and rumor propagation [16, 17]. Such approaches typically draw analogies between individual agents in the social system and particles in a corresponding physical system and then identifying macroscopically observed phenomena with microscopic agent-agent interactions [18, 19, 20].

In this spirit, we seek to tie the emergence of social structures to specific interactions between agents within a general version of the recently-introduced advancementdecline process 21, 22, 23. . In our model, the social standing of each agent is characterized by a single number, its fitness. Agents increase their fitness by interacting with other agents and also, their fitness may decline spontaneously. This simple model has only two parameters: the probability that the fitter agent advances in an interaction and the rate of decline.

We find that a rich variety of familiar social structures emerges as a result of the competition between advancement and decline. When decline dominates, the society is static and the fitness distribution approaches a steady

\footnotetext{
*Electronic address: ebn@lanl.gov

${ }^{\dagger}$ Electronic address: fvazquez@buphy.bu.edu

‡Electronic address: redner@bu.edu
}

state. When the decline rate is comparable to the advancement rate, the society is dynamic and the characteristic fitness of the population increases linearly with time. In this case, there are several possibilities. When the less fit agent benefits from social interactions, an egalitarian society arises in which all agents advance at the same rate. Consequently, inequalities among agents are small. On the other hand, when the fitter agent tends to benefit in competitions, agents advance at different rates and social inequalities increase with time. Depending on the relative influence of advancement and decline, either the entire population or only a fraction of it may be upwardly mobile. In the latter case, the society consists of a static lower class and an upwardly-mobile middle class.

In Section [II] we introduce the general advancementdecline process and the governing master equations. The overall class structure and the statistics of the mobile middle class are obtained using scaling analysis in Section III The basic features of the egalitarian society are investigated in section IV where the cumulative fitness distribution may be largely determined by linear traveling wave analysis. In Section $\nabla$ the statistics of the lower class, where the fitness distribution is steady, are determined. We conclude in section VI

\section{THE ADVANCEMENT-DECLINE MODEL}

We model a scenario in which the social status of an agent benefits from increased social interactions, while solitude or isolation have the opposite effect. Indeed, highly connected individuals often have better access to information, resources, and power, that are often gained as a result of social interactions. Thus, in our model there are two competing evolutionary processes that influence the fitness of agents: (i) advancement via social interactions, and (ii) decline due to the lack of interactions (Fig. (1). For simplicity, social standing is represented by 
a single parameter, the integer-valued fitness $k \geq 0$.

(i) Advancement. Agents interact in pairs, and as a result of the interaction, only one advances. There are two possibilities: either the fitter agent advances or the less fit advances. We allow the fitter agent to advance with probability $p$ and the less fit agent to advance with probability $1-p$. Thus, when two agents with fitness $k$ and fitness $j$ interact (with $k>j$ ), the outcome is

$$
\begin{array}{ll}
(k, j) \rightarrow(k+1, j) & \text { with probability } p \\
(k, j) \rightarrow(k, j+1) & \text { with probability } 1-p .
\end{array}
$$

For $p=1$ the fitter agent always advances 23], while for $p=0$ the less fit agent always advances. The interaction rules are defined so that one randomly-chosen agent advances when two equally-fit agents interact. Without loss of generality, the interaction rate is set to $1 / 2$. Also, we consider the thermodynamic limit where the number of agents is infinite.

(ii) Decline. In the decline step, the fitness of an individual decreases according to

$$
k \rightarrow k-1
$$

with rate $r$. This process reflects a natural tendency for fitness to decrease in the absence of social activity. We impose the lower limit for fitness to be $k=0$; once an individual reaches zero fitness, there is no further decline.

Our goal is to understand how the fitness distribution of a population evolves as a function of the two model parameters, the advancement probability $p$ and the decline rate $r$. Let $f_{k}(t)$ be the fraction of agents with fitness $k$ at time $t$. In the mean-field limit, where any pair of agents is equally likely to interact, the fitness distribution
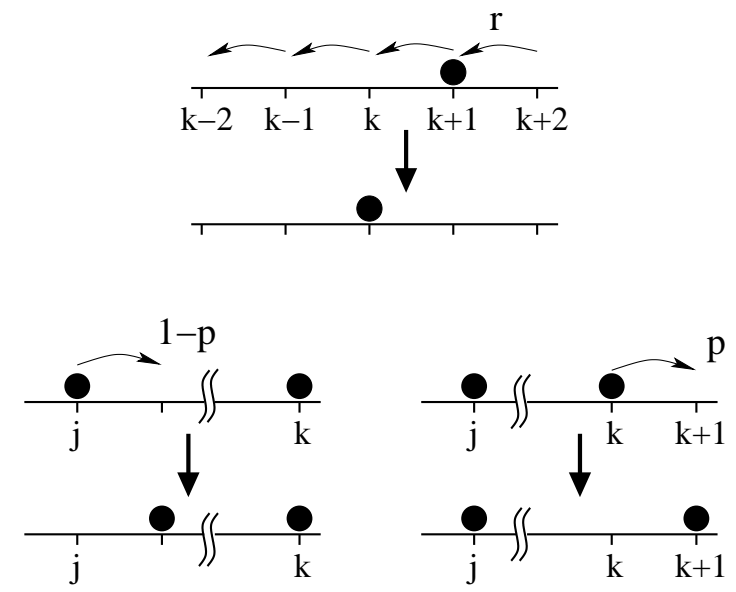

FIG. 1: The elemental processes of the advancement-decline model. Top: the decline process. Bottom: the advancement process, with either the fitter agent advancing (right) or the less fit agent advancing (left). obeys the master equation

$$
\begin{aligned}
\frac{d f_{k}}{d t} & =r\left(f_{k+1}-f_{k}\right)+p\left(f_{k-1} F_{k-1}-f_{k} F_{k}\right) \\
& +(1-p)\left(f_{k-1} G_{k-1}-f_{k} G_{k}\right)+\frac{1}{2}\left(f_{k-1}^{2}-f_{k}^{2}\right) .
\end{aligned}
$$

Here $F_{k}=\sum_{j=0}^{k-1} f_{j}$ and $G_{k}=\sum_{j=k+1}^{\infty} f_{j}$ are the respective cumulative distributions of agents with fitness less than $k$ and fitness greater than $k$. The boundary condition is $f_{-1}(t)=0$. The first pair of terms accounts for decline, the second pair of terms describes interactions where the stronger agent advances, and the third pair of terms accounts for interactions where the weaker agent advances. The last pair of terms describes interactions between two equal agents and it reflects that when two such agents interact, only one of them advances. The prefactor $1 / 2$ arises because there are half as many ways to chose equal agents as there are for different agents. We consider the initial condition where all agents have the minimal fitness $f_{k}(0)=\delta_{k, 0}$.

It proves useful to rewrite the evolution equation in a closed form that involves only the cumulative distribution. Summing the rate equations (11) and using the relations $f_{k}=F_{k+1}-F_{k}$ and $G_{k}=1-F_{k+1}$, the cumulative distribution $F_{k}$ obeys

$$
\begin{aligned}
\frac{d F_{k}}{d t} & =r\left(F_{k+1}-F_{k}\right)+p F_{k-1}\left(F_{k-1}-F_{k}\right) \\
& +(1-p)\left(1-F_{k}\right)\left(F_{k-1}-F_{k}\right)-\frac{1}{2}\left(F_{k}-F_{k-1}\right)^{2} .
\end{aligned}
$$

The boundary conditions are $F_{0}=0, F_{\infty}=1$, and the initial condition is $F_{k}(0)=1$ for $k \geq 1$. There is a one-toone correspondence between the four terms in equations (11) and (2). The master equation for the cumulative distribution can be simplified by consolidating the advancement terms

$$
\begin{aligned}
\frac{d F_{k}}{d t} & =r\left(F_{k+1}-F_{k}\right)+(1-p)\left(F_{k-1}-F_{k}\right) \\
& +(p-1 / 2)\left(F_{k-1}^{2}-F_{k}^{2}\right) .
\end{aligned}
$$

The mean fitness $\langle k\rangle=\sum_{k} k f_{k}$ evolves according to

$$
\frac{d\langle k\rangle}{d t}=\frac{1}{2}-r\left(1-f_{0}\right)
$$

This result can be derived directly by summing the master equations (11) or even simpler, from the definition of the advancement-decline process. The first term accounts for advancement, where interactions occur with rate $1 / 2$ such that each interaction advances only one agent. The second term stems from decline and reflects the fact that all agents except for the least-fit ones decline with rate $r$.

We now discuss the basic social structures that emerge from the solution to the master equation. 


\section{EMERGENCE OF SOCIAL STRUCTURES}

\section{A. Scaling solution}

We determine the class structure of the population via a simple scaling analysis of the master equation. Let us take the continuum limit of the master equation by replacing differences with derivatives, $F_{k+1}-F_{k} \rightarrow \partial F / \partial k$. To first order in this "spatial" derivative, we obtain the nonlinear partial differential equation

$$
\frac{\partial F}{\partial t}=[p+r-1-(2 p-1) F] \frac{\partial F}{\partial k} .
$$

When the spatial derivative and the temporal derivative balance, the typical fitness increases linearly with time, $k \sim t$. Therefore, we make the scaling ansatz

$$
F_{k}(t) \simeq \Phi\left(\frac{k}{t}\right)
$$

The boundary conditions are $\Phi(0)=0$ and $\Phi(\infty)=1$.

Substituting this scaling form in Eq. (5), the partialdifferential equation reduces to the ordinary differential equation

$$
[(p+r-1+x)-(2 p-1) \Phi(x)] \frac{d \Phi}{d x}=0,
$$

where the prime denotes differentiation with respect to the scaling variable $x \equiv k / t$. The solution is either $d \Phi / d x=0$, i.e.,

$$
\Phi(x)=\text { constant }
$$

or the linear function

$$
\Phi(x)=\frac{p+r-1}{2 p-1}+\frac{x}{2 p-1} .
$$

Using these two solutions and invoking (i) the boundary conditions $\Phi(0)=0$ and $\Phi(\infty)=1$, (ii) the bounds $0<\Phi(x)<1$, (iii) monotonicity of the cumulative distribution, $d \Phi(x) / d x \geq 0$, and (iv) the assumption that the scaling function changes continuously with $p$ and $r$, we can then deduce the four possible social structures of the population.

1. Middle-Class Society: We first examine the conditions for the linear scaling function (9) to apply. First, the cumulative scaling function (6) must be a monotonically increasing function. Therefore, the linear solution (9) holds only when its slope is positive, that is, when $p>1 / 2$. Second, the scaling function is bounded, $0 \leq \Phi(x) \leq 1$; this condition implies the lower and upper bounds

$$
x_{-}=1-(p+r) \quad \text { and } \quad x_{+}=p-r
$$

on the scaled fitness. The obvious constraints $x_{-}>0$ and $x_{+}>0$ lead to the conditions $p+r<1$ and $p>r$. By imposing continuity, as well as the limiting behaviors $\Phi(x)=0$ and $\Phi(x)=1$ outside the linear region,

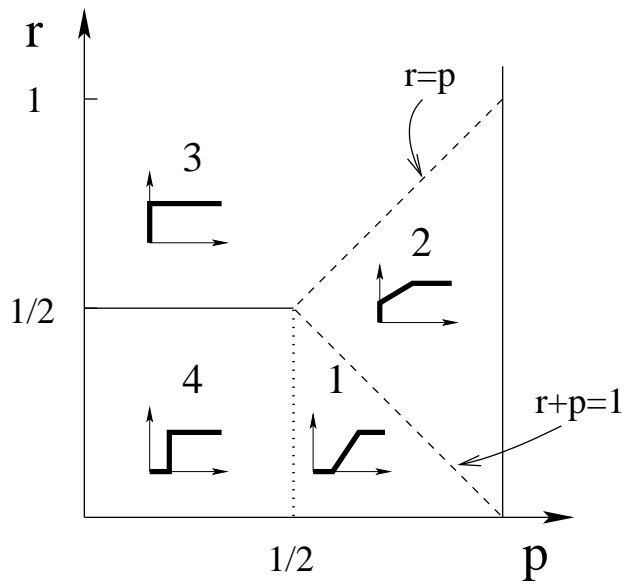

FIG. 2: Phase diagram of the advancement-decline model. The small graphs in each region are sketches of the scaled cumulative fitness distribution.

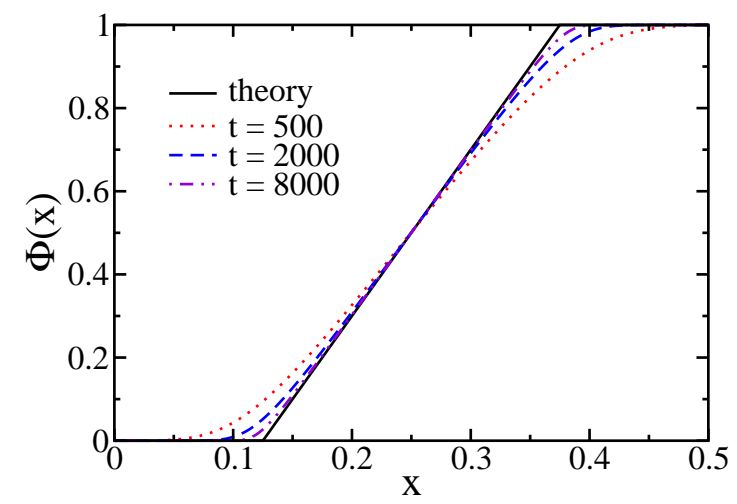

FIG. 3: Middle-Class society. The scaled cumulative fitness distribution $\Phi(x)$ versus $x=k / t$ at different times for $p=5 / 8$ and $r=1 / 4$.

the scaled cumulative distribution is the piecewise linear function (Fig. 3):

$$
\Phi_{\mathrm{M}}(x)= \begin{cases}0 & 0<x<x_{-} \\ \frac{p+r-1}{2 p-1}+\frac{x}{2 p-1} & x_{-}<x<x_{+} \\ 1 & x_{+}<x\end{cases}
$$

This behavior describes a middle class society where all agents are upwardly mobile, as their fitness improves linearly with time. In this case, social inequalities also increase indefinitely with time: the agents at the bottom of the middle class have fitness $k_{-}=[1-(p+r)] t$ and the richest agents have fitness $k_{+}=(p-r) t$. The middleclass society lies within the triangular region defined by the lines $r+p=1, r=0$, and $p=1 / 2$, shown in Fig. 2 .

2. Hierarchical Society: Along the line $r+p=1$, the fitness of the poorest agents vanishes. Moreover, the linear scaling solution (9) has a finite positive value at zero fitness for a range of parameter values $p$ and $r$. These two observations suggest the existence of another type 


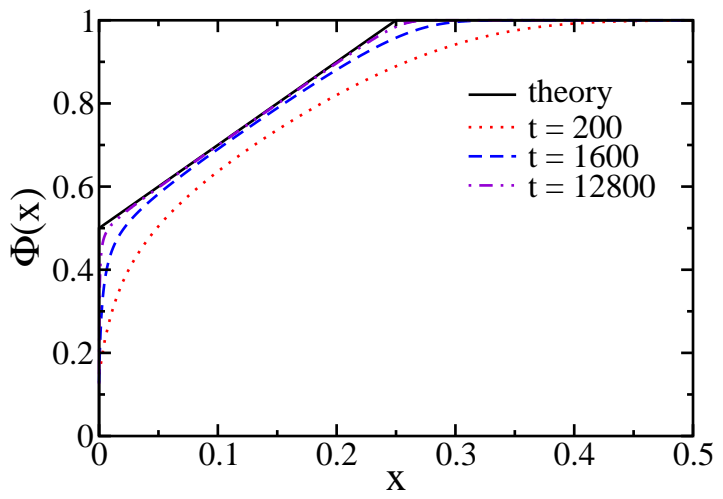

FIG. 4: Hierarchical society. The scaled cumulative fitness distribution $\Phi(x)$ versus $x=k / t$ at different times for $p=3 / 4$ and $r=1 / 2$.

of piecewise linear solution with $\Phi(0)>0$. The bounds $0<\Phi(0)<1$ impose the conditions $p+r>1$ and $r>p$. In this region, the scaling function has two distinct components (Fig. 4)

$$
\Phi_{\mathrm{H}}(x)= \begin{cases}\frac{p+r-1}{2 p-1}+\frac{x}{2 p-1} & 0<x<x_{+} \\ 1 & x_{+}<x .\end{cases}
$$

Thus, we find a hierarchical society (Fig. 2) that includes both an upwardly-mobile middle class and a static lower class. The lower class consists of a finite fraction

$$
L=\frac{p+r-1}{2 p-1}
$$

of agents with zero fitness (in scaled units). In section $\nabla$ we examine the lower class more closely and show that its fitness distribution is time-independent and extends only over a finite range.

3. Lower-Class Society: When the fraction $L$ of agents with zero fitness reaches 1 , the entire population is poor. For $p>1 / 2$, the condition $L=1$ occurs on the boundary $p=r$. At this point the fitness distribution becomes a step function,

$$
\Phi_{\mathrm{L}}(x)=\Theta(x),
$$

with $\Theta(x)=0$ for $x \leq 0$ and $\Theta(x)=1$ for $x>0$. We therefore conclude that there is a region of the phase diagram where the scaled fitness of the entire population is zero. For any initial state, the fitness distribution quickly approaches the step-function in a lower-class society.

4. Egalitarian Society: There is another region of the phase diagram where the fitness distribution also becomes a step function. When $p=1 / 2$ and $r<1 / 2$, then both $x_{+}$and $x_{-}$are equal to $1 / 2-r$. Therefore $\Phi(x)=\Theta(x-v)$ with $v=1 / 2-r$. Since the scaling function must change in a continuous fashion, we conclude that for $p<1 / 2$, the scaling function is again a step function but with a jump at non-zero fitness. That is

$$
\Phi_{\mathrm{E}}(x)=\Theta(x-v) .
$$

In this egalitarian society, all agents have the same scaled fitness $x=v$ or alternatively the fitness $k \approx v t$. The velocity $v$ follows easily from the average fitness (4). Since all agents advance at constant rate, then the term $-r f_{0}$ is negligible and therefore, the propagation velocity is

$$
v=\frac{1}{2}-r
$$

In section IV we show that in this society, the fitness differences between agents are small and do not grow with time. This is the sense in which the society is egalitarian. When $p<1 / 2$, the weaker agent preferentially benefits in an interaction, so that the rich effectively supports the poor. We also note that the lower class and the egalitarian society share one common feature: they do not have a middle class. The boundary between these two phases, determined by the condition $v=0$, is the line $r=1 / 2$ (Fig. 2).

Our numerical integration of the evolution equations confirms the overall picture of four different social structures (Fig. 2): a middle class society (Fig. 3), a hierarchical society (Fig. 4), a lower-class society as in (14), and an egalitarian society as in (15). The numerical data was obtained by integrating $F_{k}$ for $0 \leq k<10000$ using a fourth-order Adams-Bashforth method [24], with a specified accuracy of $10^{-10}$ in the distribution $F_{k}$.

\section{B. Refinements to the Scaling Solutions}

Our numerical results for the cumulative distribution $F_{k}$, when plotted versus the scaling variable $x=k / t$, smoothly approaches the appropriate expressions for the piecewise linear scaling function $\Phi(x)$ derived in the previous subsection (Figs. 3 \& 4). As time increases, the fitness distribution narrows. The simulations also show that the approach to the scaling solution is slowest in the vicinity of the extremes of the middle class $x=x_{-}$and $x=x_{+}$(with $x_{-}=0$ for the hierarchical phase).

The correction to scaling near these extrema can be determined by keeping derivatives up to second order in the continuum limit of the master equation. This approximation gives the nonlinear diffusion equation [25, 26

$$
\begin{aligned}
\frac{\partial F}{\partial t} & =(r+p-1) \frac{\partial F}{\partial k}+\frac{1}{2}(1+r-p) \frac{\partial^{2} F}{\partial^{2} k} \\
& +(1-2 p) F\left[\frac{\partial F}{\partial k}-\frac{1}{2} \frac{\partial^{2} F}{\partial^{2} k}\right]+(p-1 / 2)\left(\frac{\partial F}{\partial k}\right)^{2}
\end{aligned}
$$

The linear terms are separately displayed in the first line and the nonlinear terms in the second.

Let us first consider the poorest agents, i.e, the behavior close to $x=x_{-}$. Since the cumulative fitness 
distribution is small near this point, the nonlinear terms can be neglected and the governing equation (17) reduces to the standard convection-diffusion equation

$$
\frac{\partial F}{\partial t}+v_{-} \frac{\partial F}{\partial k}=D_{-} \frac{\partial^{2} F}{\partial^{2} k}
$$

with propagation velocity $v_{-}=x_{-}=1-p-r$ and diffusion coefficient $D_{-}=(1-p+r) / 2$. Indeed, since the fitness distribution is obtained from the cumulative distribution by differentiation, $f=\partial F / \partial k$, the fitness distribution satisfies the same equation (5) as the cumulative distribution.

For the middle-class society, we therefore conclude that the bottom of the middle class has a Gaussian tail, with the center of the Gaussian located at $k_{-}=v_{-} t$ and with width $\sqrt{D_{-} t}$. The same analysis can be carried out for the hierarchical society, where the quantity $F-L$ now satisfies the diffusion equation with zero velocity $v_{-}=0$ and diffusivity $D_{-}=r$. Conversely, the distribution for the top end of the middle class can be obtained by analyzing $1-F$. It is immediate to show that this quantity again obeys Eq. (18) with velocity $v_{+}=x_{+}=p-r$ and diffusivity $D_{+}=(r+p) / 2$. We conclude that the extremes of the middle class are characterized by Gaussian tails whose extents grow diffusively with time. In terms of the scaling variable $x$, the deviation from the scaling function $\Phi(x)$ is appreciable only within a region of whose width is shrinking as $t^{-1 / 2}$.

For the special case $p=1 / 2$, the nonlinear terms vanish and the fitness distribution is described exactly by the linear convection-diffusion equation (18) with drift velocity $v=1 / 2-r$ and diffusion coefficient $D=(r+p) / 2$ (the nonlinear term is negligible). Thus there is a drift toward smaller fitness for $r>1 / 2$ and the fitness distribution approaches a steady-state profile that decays exponentially with fitness. In the opposite case of $v>0$, the fitness distribution is simply a Gaussian that drifts to larger fitness with velocity $\frac{1}{2}-r$ and whose width is proportional to $\sqrt{D t}$. In the case of $p=1 / 2$, the relative position of an agent in the society is irrelevant and advancement reduces to a pure random walk [27].

\section{EGALITARIAN SOCIETY}

In the egalitarian phase, the step function form of the scaling solution, Eq. (15), suggests that the fitness distribution has the traveling wave form

$$
F_{k}(t) \rightarrow U(k-v t)
$$

with the propagation velocity (16). This is confirmed by numerical integration of the master equation (3), as shown in Fig. (5).

To determine the shape of the wave $U(z)$ analytically, we substitute the waveform (19) into the master equation (3) to give the nonlinear difference-differential equation

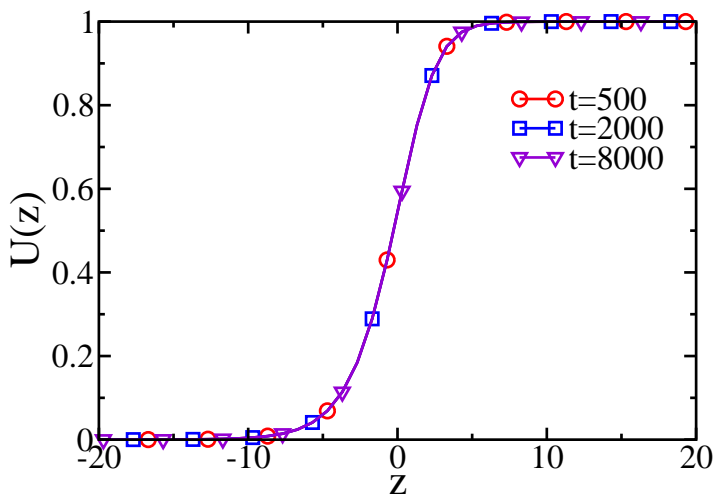

FIG. 5: The scaled cumulative fitness distribution $U(z)$ versus $z=k-v t$, where $v=1 / 2-r$ is the speed of wavefront at different times for $p=1 / 4$ and $r=1 / 4$ (egalitarian society).

for $U(z)$

$$
\begin{aligned}
-v U^{\prime}(z) & =r[U(z+1)-U(z)]+(1-p)[U(z-1)-U(z)] \\
& +(p-1 / 2)\left[U^{2}(z-1)-U^{2}(z)\right] .
\end{aligned}
$$

The boundary conditions are $U(-\infty)=0$ and $U(\infty)=1$.

\section{A. Waveforms in the tail regions}

We apply standard linear analysis in the tail regions to deduce the leading and trailing shapes of the waveform. When $z \rightarrow-\infty$, then $U \ll 1$ and therefore $U^{2} \ll U$. To first order in $U$, Eq. (20) becomes

$v U^{\prime}+r[U(z+1)-U(z)]+(1-p)[U(z-1)-U(z)]=0$.

The behavior in this case is determined by the balance between decline and advancement events where the less fit agent advances. The solution to this linearized equation is the exponential decay

$$
U(z) \sim e^{\alpha z}, \quad z \rightarrow-\infty .
$$

Substituting this form and (16) into the linearized equation, the decay constant $\alpha$ is the root of the following equation

$$
1 / 2-r=\alpha^{-1}\left[(1-p)\left(1-e^{-\alpha}\right)-r\left(e^{\alpha}-1\right)\right] .
$$

Similarly, in the limit $z \rightarrow \infty$ we linearize the wave equation (20) for the small quantity $R=1-U$ to obtain

$$
v R^{\prime}=r[R(z)-R(z+1)]+p[R(z)-R(z-1)] .
$$

In this case the behavior at large fitness is governed by the balance between decline and advancement events where the fitter agent advances 27]. The solution to the above differential equation is again the exponential decay

$$
R(z) \sim e^{-\beta z}, \quad z \rightarrow \infty
$$




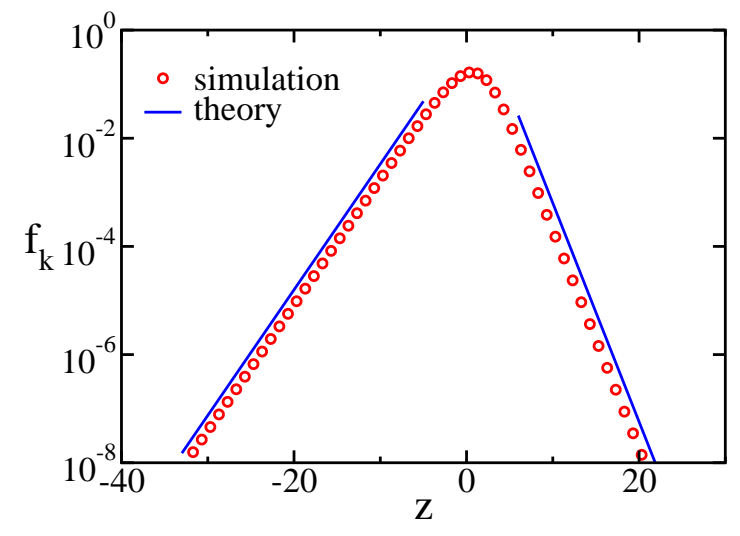

FIG. 6: Tails of the fitness distribution $f_{k}$ versus $z=k-v t$ for $p=r=1 / 4$. The theoretical predictions (21) - 24 with $\alpha=0.535572$ and $\beta=0.930821$ are indicated by straight lines.

with the decay constant $\beta$ satisfying

$$
1 / 2-r=\beta^{-1}\left[p\left(e^{\beta}-1\right)-r\left(1-e^{-\beta}\right)\right] .
$$

We conclude that the likelihood of having agents that are much richer or much poorer than the average fitness $k=v t$ in the egalitarian society is exponentially small, as illustrated in Fig. 6 The society therefore consists of agents whose fitnesses are all roughly the same, $k \approx v t$. As one might naturally anticipate, social inequalities are small under the dynamics in which the rich preferentially gives to the poor.

\section{B. Less fit advances $(p=0)$}

For the case where the less fit agent always advances, the fitness distribution has a special form. In this case, the complementary cumulative distribution obeys

$$
\frac{d G_{k}}{d t}=r\left(G_{k+1}-G_{k}\right)+\frac{1}{2}\left(G_{k-1}^{2}-G_{k}^{2}\right)
$$

with the initial condition $G_{k}(0)=\delta_{k,-1}$ and the boundary condition $G_{-1}(t)=1$.

We expect that the fitness distribution will continue to have the form of a propagating wave. Substituting the traveling wave form $G_{k}(t) \rightarrow R(k-v t)$ into the master equation (25) gives

$$
-v R^{\prime}(z)=r[R(z+1)-R(z)]+\frac{1}{2}\left[R^{2}(z-1)-R^{2}(z)\right] .
$$

An exponential solution does not give asymptotic balance of terms as $z \rightarrow \infty$, and we therefore attempt a solution of the form $R(z) \sim \psi(z) e^{-\phi(z)}$. Substituting this form into the above equation and keeping only the dominant term $\frac{1}{2} R^{2}(z-1)$ on the right-hand-side gives

$$
v \psi(z) \phi^{\prime}(z) e^{-\phi(z)} \approx \frac{1}{2} \psi^{2}(z-1) e^{-2 \phi(z-1)} .
$$

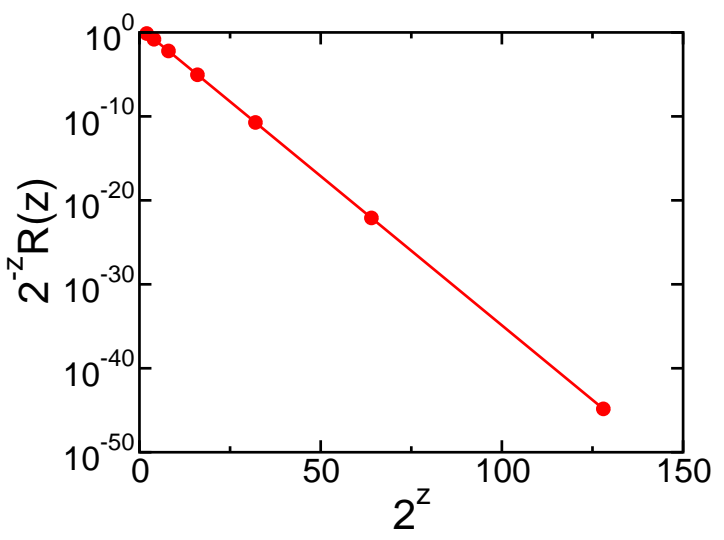

FIG. 7: The super-exponential tail: the quantity $2^{-z} R(z)$ versus $2^{z}$, obtained by numerical integration of (25) to time $t=100$ with $r=0$.

For the positive terms on the left and the right hand side to balance, the dominant exponential terms must first balance, yielding the recursion equation $\phi(z)=2 \phi(z-1)$. The solution is the exponential $\phi(z)=C 2^{z}$. Balancing the prefactors, $v \psi(z) \phi^{\prime}(z)=\frac{1}{2} \psi^{2}(z-1)$ yields $\psi(z)=$ $8 \ln 2 C v 2^{z}$. As a result, the decay in the tail region is super-exponential [28]

$$
R(z) \sim 8 \ln 2 C v 2^{z} \exp \left(-C 2^{z}\right),
$$

as $z \rightarrow \infty$. The constants $C$ and $v$ should be determined numerically. Hence, the front of the traveling wave is extremely sharp. This tail characterizes statistics of the rich, so when the rich never benefits from interactions with the poor, rich agents are ultra-rare (Fig. 17). Even though the leading tail extends to only a handful of sites, it is still possible to verify the super-exponential decay (27). In contrast, the $z \rightarrow-\infty$ tail that characterizes the poor is not altered; it has the same exponential tail as in Eq. (21).

\section{THE LOWER CLASS}

We now determine the fitness distribution of poorest agents, a class that exists in both the hierarchical and lower-class societies. As we shall now show, the fitness distribution of the lower class in the limit of small but non-zero fitness approaches a steady state. For the case of the hierarchical society, we write $F_{k}=L\left(1-g_{k}\right)$, where $L$ is the lower-class fraction, with the deviation $g_{k}$ vanishing for large $k$. Substituting this form into the master equation (31) and setting the time derivative to zero, gives

$$
r \frac{g_{k}-g_{k+1}}{g_{k-1}-g_{k}}=1-p+(p-1 / 2) L\left(2-g_{k}-g_{k-1}\right) .
$$

Consider first the lower-class society, for which the entire population is poor, $L=1$. Using this fact, and 
neglecting terms of the order $g^{2}$, we find

$$
\frac{g_{k}-g_{k+1}}{g_{k-1}-g_{k}}=\frac{p}{r} \text {. }
$$

The solution to this equation is simply the exponential form $g_{k} \sim \alpha^{k}$ with $\alpha=p / r$. Since $f_{k}=F_{k+1}-F_{k}=$ $g_{k}-g_{k+1}$, then

$$
f_{k} \propto\left(\frac{p}{r}\right)^{k}
$$

In the lower-class society, the fitness is confined to a very small range. Notice also that this exponential decay coincides with the traveling wave solution (23) with $v$ set equal to zero, as the decay function is now $e^{-\beta}=p / r$.

Finally, we consider the hierarchical society. Using (13) and following the same steps that led to Eq. (29), we obtain

$$
\frac{g_{k}-g_{k+1}}{g_{k-1}-g_{k}}=1-\gamma g_{k}
$$

with $\gamma=(r+p-1) / r$. To determine $g_{k}$, we expand the differences to second order and assume that $g^{\prime \prime} \ll$ $g^{\prime}$ to give, after straightforward steps, $g^{\prime \prime}+\gamma g g^{\prime}=0$. The asymptotic solution to this equation is $g \simeq 2 /(\gamma k)$. Finally, using $f_{k}=F_{k+1}-F_{k}$ and $F_{k}=L\left(1-g_{k}\right)$, we determine the fitness distribution from $f_{k} \simeq-L g^{\prime}$ to be

$$
f_{k} \simeq \frac{2 r}{2 p-1} k^{-2} .
$$

Thus, for the hierarchical society, the fitness distribution has a power-law large-fitness tail in the lower class region (see also [23] for more details).

As discussed in section [II] there is a diffusive boundary layer that separates the steady-state fitness distribution in the lower class and time dependent fitness distribution in the middle class. From Eqs. (6) and (12), the fitness distribution in the middle class is $f_{k} \simeq[(2 p-1) t]^{-1}$. Equating this expression with Eq. (32) gives a crossover scale

$$
k_{*} \simeq \sqrt{2 r t} .
$$

Thus, the steady-state region extends over a fitness range that grows as $t^{1 / 2}$. We also note that this crossover scale agrees with the diffusivity $D_{-}=r$, obtained in section III In terms of the variable $x=k / t$, the size of this region $x_{*} \sim t^{-1 / 2}$ decays with time. Thus, a diffusive boundary layer separates the lower class and the middle class.

\section{CONCLUSIONS}

In summary, we have seen that the competition between advancement and decline leads to a rich and realistic set of possible social structures. From the master equation for the underlying fitness distribution, we obtain three types of classes: a static lower class, a mobile but disperse middle class, and a mobile but "condensed" egalitarian class. The population as a whole organizes into four types of societies, three of which consist of one of these classes, and a hierarchical society in which the lower class and the middle class coexist. Two parameters, the rate of decline and the advancement probability, quantify the competition between advancement and decline. The overall social organization is determined solely by these two parameters.

The fitness distribution has a very different character in each of the classes. In the lower class, this fitness distribution approaches a steady state. In the middle class, the distribution is self-similar in time and correspondingly the characteristic fitness increases linearly with time. Although agents are upwardly mobile, the disparities between agents in the middle class also grows indefinitely. In the egalitarian class, the fitness distribution follows a traveling wave, so that all agents constantly advance, but fitness differences between agents remains small.

Much of the richness of the phenomenology is due to the fact that the mechanisms for advancement and decline are fundamentally different. One requires interaction between agents, while the other is a single-agent process. This dichotomy is reflected by the master equation where the decline terms are linear but the advancement terms are nonlinear. As a result, there is no detailed balance and the dynamics are non-equilibrium in character.

It should be interesting to use the advancement-decline model to analyze real-world data. One natural application is to wealth and income distributions of individuals, where both power-law and exponential behavior has been observed 11, 12. A related issue is the wealth of nations. It is well documented that the wealth distribution of countries is extremely inequitable, with $60 \%$ of the world's population producing just $5.6 \%$ of the planet's gross domestic product (GDP), another $20 \%$ producing $11.7 \%$, and the remaining $20 \%$ of the population producing $82.7 \%$ of the GDP [29]. The existence of such a large underclass corresponds to a large decline rate in our diversity model and it may be worthwhile to understand the social mechanisms for such a large decline. Another possibility is sports statistics where the winning percentage distribution of teams plays the role of the fitness distribution [30].

\section{Acknowledgments}

We thank Philip Rosenau for useful discussions. We acknowledge financial support from DOE grant W-7405ENG-36 and NSF grant DMR0227670. 
[1] I. D. Chase, Amer. Sociological Rev. 45, 905 (1980).

[2] R. V. Gould, Amer. J. Sociology 107, 1143 (2002).

[3] H. G. Landau, Bull. Math. Biophys. 13, 1 (1951).

[4] E. O. Wilson, Sociobiology, (Harvard University Press, Cambridge, MA, 1975).

[5] E. O. Wilson, The Insect Societies, (Harvard University Press, Cambridge, MA, 1971).

[6] W. C. Allee, Biol. Symp. 8, 139 (1942).

[7] A. M. Guhl, Anim. Behav. 16, 219 (1968).

[8] M. W. Schein and M. H. Forman, Brit. J. Anim. Behav. 3, 45 (1955).

[9] M. Varley and D. Symmes, Behaviour 27, 54 (1966).

[10] I. D. Chase, Behav. Sci. 19, 374 (1980).

[11] S. Ispolatov, P. L. Krapivsky, and S. Redner, Eur. Phys. Jour. B 2, 267 (1998).

[12] A. Dragulescu and V. M. Yakovenko, Eur. Phys. Jour. B 17, 723 (2000).

[13] G. Weisbuch, G. Deffuant, F. Amblard, and J. P. Nadal, Complexity 7, 55 (2002).

[14] E. Ben-Naim, P. L. Krapivsky, and S. Redner, Physica D 183, 190 (2003).

[15] D. Stauffer and H. Meyer-Ortmanns, Int. J. Mod. Phys. B 15, 241 (2004).

[16] S. Wasserman and K. Faust, Social Network Analysis (Cambridge University Press, Cambridge, 1994).

[17] M. Girvan and M. E. J. Newman, Proc. Natl. Acad. Sci. USA 99, 7821 (2002).

[18] W. Weidlich, Sociodynamics: A Systematic Approach to Mathematical Modelling in the Social Sciences (Harwood
Academic Publishers, 2000)

[19] D. Helbing, I. Farkas, and T. Vicsek, Nature 407, 487 (2000).

[20] I. D. Couzin, J. Krause, N. R. Franks, S. A. Levin, Nature 433, 513 (2005).

[21] E. Bonabeau, G. Theraulaz, and J.-L. Deneubourg, Physica A 217, 373 (1995).

[22] A. O. Sousa and D. Stauffer, Intl. J. Mod. Phys. C 5, 1063 (2000); K. Malarz, D. Stauffer, and K. Kulakowski, physics/0502118.

[23] This special case was discussed in E. Ben-Naim and S. Redner, J. Stat. Mech L11002 (2005).

[24] D. Zwillinger, Handbook of Differential Equations (Academic Press, London, 1989).

[25] G. B. Whitham, Linear and Nonlinear Waves, (Wiley, New York, 1974).

[26] J. M. Burgers, The nonlinear diffusion equation (Reidel, Dordrecht, 1974).

[27] E. Ben-Naim, P. L. Krapivsky, and S. N. Majumdar, Phys. Rev. E 64, R035101 (2000).

[28] E. Ben-Naim, P. L. Krapivsky, and S. N. Majumdar, unpublished.

[29] United Nations Development Program 1992, Human Development Report (Oxford University press for the United Nations Development Program, New York, 1992).

[30] E. Ben-Naim, F. vazquez, and S. Redner, "What is the most competitive sport?", preprint. 